\title{
A Novel ABCA4 Mutation Associated with a Late-Onset Stargardt Disease Phenotype: A Hypomorphic Allele?
}

\author{
Cindy S. Kaway ${ }^{a} \quad$ Madeleine K.M. Adams ${ }^{b-d}$ Kevin Sean Jenkins ${ }^{b, c}$ \\ Christopher J. Layton ${ }^{\text {b-d }}$ \\ ${ }^{a}$ Faculdade Evangélica do Paraná, Curitiba, Brazil; ${ }^{b}$ Gallipoli Medical Research Institute, \\ Brisbane, QLD, Australia; 'University of Queensland School of Medicine, \\ Herston, QLD, Australia; ${ }^{d}$ Greenslopes Private Hospital Ophthalmology Department, \\ Greenslopes Hospital, Brisbane, QLD, Australia
}

\section{Keywords}

Stargardt disease $\cdot$ Macular dystrophy $\cdot$ ABCA4 $\cdot$ Mutation

\begin{abstract}
Background: Stargardt disease (STGD) is the most common juvenile hereditary macular dystrophy. In the majority of cases, the diagnosis is made prior to 20 years of age and usually leads to loss of central vision. Late-onset STGD affects a smaller number of patients. Identifying genetic changes which could be associated with clinically important differences in severity or presentation of the disease is important for understanding the mechanisms of visual loss and for planning future therapeutic approaches. Methods: We report a patient with the classic phenotype of STGD with late-onset mild disease exhibiting a slow clinical progression over 14 months of follow-up. Results: A 37-year-old man presented with STGD and good vision of $6 / 24$ in the right eye and of $6 / 6$ in the left eye as well as typical electrophysiology findings. Objective and subjective visual deterioration was not noted over a period of 14 months. Macular genetic testing revealed a novel missense mutation in ABCA4 (Thr829Met) combined with Gly1961Glu, a classic STGD mutation usually associated with a moderately severe phenotype. Conclusions: It is suggested that the Thr829Met mutation could give rise to a hypomorphic allele of the $A B C$ transporter with a resultant phenotype of comparatively mild STGD.

(C) 2017 The Author(s)




\section{Introduction}

Stargardt disease (STGD) represents the most common juvenile hereditary macular dystrophy, affecting approximately 1 in 10,000 individuals [1]. In the majority of cases, STGD is diagnosed within the first or second decade of life with a progressive deterioration of central vision usually leading to blindness [2]. Late-onset STGD ( $>20$ years) has become more recognized since the availability of genetic testing; it affects a smaller number of patients [3] and has a better prognosis compared to early-onset STGD [3].

Clinical manifestations of STGD are thought to be due to an accumulation of lipofuscin in the outer retina and retinal pigment epithelium, which, clinically, results in a progressive loss of central vision associated with perifoveal yellow-white flecks, a beaten-bronze appearance of the macula and eventually bilateral atrophy of the central retina [1,2]. Visual acuity can range from $6 / 6$ to $6 / 120$ [3].

Autosomal recessive STGD is usually caused by a mutation in the gene for the $A B C$ transporter (ABCR or ABCA4), which is highly expressed in the retina. ABCA4 is responsible for encoding the adenosine triphosphate-binding cassette transporter in rod outer segment discs and is important in the visual cycle, transporting the toxic all-trans retinal out of the disc for recycling by the retinal pigment epithelium [4].

We report a patient with the classic phenotype of late-onset STGD exhibiting slow clinical progression and apparently caused by a novel missense mutation in association with a classic STGD mutation.

\section{Case Presentation}

A 37-year-old man presented with a 3-year history of decreased vision in the right eye, which had recently become worse. He reported no previous visual problems and had been diagnosed with a congenital colour vision deficiency at an early age. He had never been treated with antimalarial therapy or retinotoxic drugs, had no night vision symptoms and enjoyed a normal diet. He reported trauma to his head in a soccer game, and subsequent investigation had revealed an apparently incidental colloid cyst of the third ventricle measuring $6 \mathrm{~mm}$. He denied a history of consanguinity in his family background. His family, based in the Middle East, had no history of inherited retinal disease, poor vision or colour vision disturbance; however, his father had recently been diagnosed with chronic central serous retinopathy at a late age, and a facsimile of an otherwise normal fluorescein angiogram with no signs for choroidal darkening or hyperfluorescent flecks was available from this recent assessment. Apparently, his condition had improved significantly with systemic spironolactone treatment.

Examination revealed a visual acuity of $6 / 24$ in the right eye and of $6 / 6$ in the left eye, normal pupil reactions, $1 / 15$ Ishihara colour vision test plates read correctly with each eye, full ocular motility and an otherwise normal cranial nerve examination. Anterior segment examination showed no abnormalities, and fundus examination (Fig. 1a, b) revealed scattered atrophy and pisciform fundal flecks at both maculae, which were worse in the right eye. Fluorescein angiography (Fig. 1c, d) demonstrated a silent choroid and partial bull's eye maculopathy which was worse in the right eye. Optical coherence tomography (OCT) (Fig. $1 \mathrm{e}-\mathrm{h}$ ) showed loss of photoreceptors at both maculae with partial central sparing in the left eye. Photopic and scotopic flash electroretinograms (ERGs) showed reduced amplitudes of responses in the major ERG components in the right eye and amplitudes within the lower 
range of normal in the left eye. Implicit times in both eyes were within normal limits. The pattern ERG and multifocal ERG demonstrated central retinal dysfunction with the retention of peripheral function. Next-generation sequencing analysis with the Oxford Genetics Testing Laboratory Macular Gene Panel revealed 2 mutations in the ABCA4 genes: c.5882G>A p.(Gly1961Glu), a common ABCA4 mutation, and c.2486C $>$ T p.(Thr829Met), which has not been previously reported in association with a diagnosis of STGD. As the subject's parents were unavailable for segmentation analysis, there remains a possibility that the mutations were on the same allele.

The patient was referred for genetic counselling and underwent follow-up for a period of 14 months, maintaining baseline objective and subjective levels of vision in each eye with no change in fundal appearance or OCT.

\section{Discussion}

A spectrum of retinal phenotypes caused by ABCA4 variants can be identified through clinical observations, and over 600 disease-associated ABCA4 alleles have been reported [4]. Most have been reported only once with the most frequent disease-associated variant being described in $10 \%$ of the patients with STGD [4]. Therefore, it is important to report rare mutations, which may influence mechanisms of pathogenicity, and their correlation with genotype/phenotype.

The patient in this study carries 2 missense heterozygous mutations, Gly1961Glu and Thr829Met. The variant Gly1961Glu (c.5882G>A) is one of the most common ABCA4 mutations, especially in patients with Italian ancestry, with a frequency of $21 \%$ in these populations [5]. This mutation is usually associated with a moderate severity of the disease [6]; however, significant phenotypic variability has been reported, and there is speculation about a genotype-phenotype correlation, in which the phenotype of STGD relies on the mutation severity of the fellow allele [6].

The mechanism of cellular damage caused by the novel variant Thr829Met (c.2486C > T) is uncertain. The Thr829Met missense mutation has previously been reported in a single patient of Turkish background with the phenotype of autosomal recessive retinitis pigmentosa [7] and has never been identified in association with the STGD phenotype. Given the late onset in the presented case together with the excellent retained vision in the left eye and lack of measurable progression over 14 months of follow-up, it is suggested that the Thr829Met mutation could give rise to a hypomorphic allele of the ABC transporter with a resultant phenotype of comparatively mild STGD.

In summary, we report a classic phenotype of STGD with slow progression associated with a classic STGD mutation and a novel missense mutation. Our findings add to the understanding of disease-causing mutations of ABCA4 and their correlation with disease genotype/phenotype.

\section{Acknowledgements}

C.S.K. was support by a Science without Borders Scholarship from the Brazilian Government. 
Kaway et al.: A Novel ABCA4 Mutation Associated with a Late-Onset Stargardt Disease Phenotype: A Hypomorphic Allele?

\section{Statement of Ethics}

The authors have no ethical conflicts to disclose.

\section{Disclosure Statement}

The authors have no conflicts of interest to disclose.

\section{References}

1 Blacharski P: Fundus flavimaculatus; in Newsome DA (ed): Retinal Dystrophies and Degenerations. New York, Raven Press, 1988, pp 135-159.

-2 Stargardt K: Über familiäre, progressive Degeneration in der Maculagegend des Auges. Graefes Arch Clin Exp Ophthalmol 1909;71:534-550.

-3 Rotenstreich Y, Fishman GA, Anderson RJ: Visual acuity loss and clinical observations in a large series of patients with Stargardt disease. Ophthalmology 2003;110:1151-1158.

4 Allikmets R, et al: A photoreceptor cell-specific ATP-binding transporter gene (ABCR) is mutated in recessive Stargardt macular dystrophy. Nat Genet 1997;15:236.

5 Passerini I, et al: Novel mutations in of the ABCR gene in Italian patients with Stargardt disease. Eye 2010;24:158-164.

-6 Maugeri A, et al: The ABCA4 2588G>C Stargardt mutation: single origin and increasing frequency from South-West to North-East Europe. Eur J Hum Genet 2002;10:197-203.

-7 Özgü RK, et al: Molecular analysis of the ABCA4 gene in Turkish patients with Stargardt disease and retinitis pigmentosa. Hum Mutat 2004;23:523-523. 


\section{Case Reports in Ophthalmology}

www.karger.com/cop

Kaway et al:: A Novel ABCA4 Mutation Associated with a Late-Onset Stargardt Disease Phenotype: A Hypomorphic Allele?
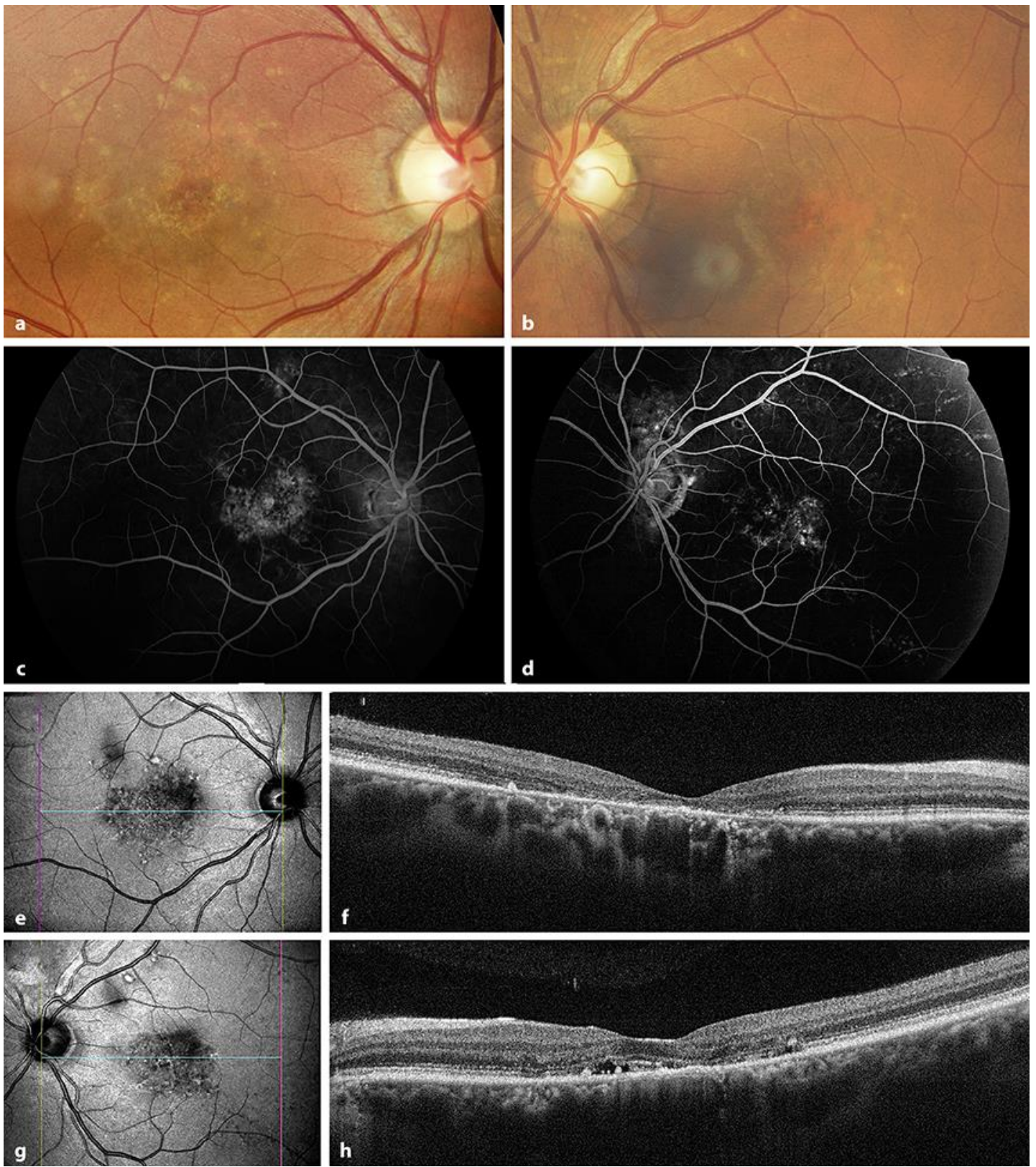

Fig. 1. Examination findings in a 37-year-old man with a 3-year history of decreased vision in the right eye. a, b Fundus photographs of the right and left eye showing retinal flecks, scattered macular atrophy and partial bull's eye maculopathy which is worse in the right eye. c, $\mathbf{d}$ Fluorescein angiogram of the right and left eye revealing a "silent" choroid, hyperfluorescent retinal flecks and partial bull's eye maculopathy which is worse in the right eye. e, f Right-eye OCT showing loss of outer retinal layers and photoreceptors. $\mathbf{g}, \mathbf{h}$ Left-eye OCT illustrating loss of outer retinal layers and photoreceptors with partial central sparing. 